\title{
Evaluation of flow rate accuracy and pressure measurements of testing rig for neurological valve hydrodynamic tests
}

\author{
José Ricardo Camilo Pinto*, Angelo Luiz Maset, José Ricardo Andrade, Bruna Monieli Mancini, \\ Geovânia Marquini Laurentino Pereira, Ricardo César Barbosa, João Manuel Domingos de Almeida Rollo
}

\begin{abstract}
Introduction: Increased production and disorders in the circulation or absorption of cerebrospinal fluid (CSF) leads to increased intracranial pressure and causes a condition called hydrocephalus. The most common treatment for this disorder is the implantation of a ventricle-peritoneal shunt, which consists of the insertion of a ventricular catheter in the cerebral ventricle, a neurological valve for drainage control and a peritoneal catheter that leads the CSF to the abdominal region. The International Organization for Standardization (ISO) 7197 introduced requirements for the design and production of the shunts and neurological valve, including in vitro hydrodynamic tests, which are indispensable for evaluating the performance of the CSF drainage systems. Methods: A testing rig built in accordance with ISO 7197 was evaluated for the uncertainty of flow and opening and closing pressure measurements, the pressure-flow relationship and reflux neurological valve resistance. The infusion pump and pressure measurement system were evaluated for calibration, hysteresis behavior and stability to determine the experimental uncertainties from hydrodynamic testing of two commercial products. Results: The equipment showed uncertainties of less than $1 \%$ in the infusion pump, less than $4 \%$ in the pressure sensor and adequate performance for the hydrodynamic tests. Conclusion: The hydrodynamic test rig allowed the opening and closing pressures and the resistance to reflux to be obtained, and it also allowed the determination of the pressure and flow rate characteristics of two neurological commercial valves with experimental uncertainties that do not impact the results and the evaluation of in vitro performance of a shunt.
\end{abstract}

Keywords Neurological valve, Cerebrospinal fluid, Shunt, Testing rig.

\section{Introduction}

Cerebrospinal fluid (CSF) is a transparent fluid with density (Wood, 1980) and viscosity (Czosnyka et al., 1997; Lavinio et al., 2008) similar to those of water and is found in the interior of the human skull and the spinal column. This fluid is produced in the choroid plexuses through a filtration mechanism similar to that of renal glomeruli (Schimer, 1995), and it exits the central nervous system through holes covering the spinal cord and is absorbed in the brain by specialized veins that take up the spinal fluid in the bloodstream. Pople (2002) and Sotelo et al. (2001) reported CSF production of approximately $500 \mathrm{ml}$ per day, i.e., of $20.8 \mathrm{ml} / \mathrm{h}$; According to Penn et al. (2011), the ventricular volume in healthy people is $33 \pm 9.4 \mathrm{ml}$. Any increase in the volume of the ventricles causes cranial hypertension.

Increased production and disturbances in the circulation or absorption of CSF due to congenital factors, meningitis, premature birth, spina bifida or tumors cause a condition called hydrocephalus (Schimer, 1995). The accumulation of fluid in the ventricles leads to increased pressure within the cranial cavity. In newborns and small children, the head will increase excessively in size because the cranial sutures have not yet closed, and in adults, there will be an increase in the cephalic perimeter (Aschoff et al., 1999).

The most common treatment for hydrocephalus is the use of a ventricle-peritoneal shunt (VPS), which consists of an implant composed of the following: a ventricular catheter, inserted inside the ventricle; a neurological valve to control the drainage of CSF; and a peritoneal catheter, which directs the fluid to the abdominal region.

A neurological valve must meet International Organization for Standardization (ISO) 7197 (International..., 2006), which describes the requirements for the development, fabrication and in vitro performance evaluation of shunts and components for hydrocephalus. The standard covers terms and definitions as well as general and specific requirements to be met by these products, such as: the guaranteed biocompatibility of the items present in the shunt and their resistance to leaks; the presence of radiopaque 
markings to identify the working pressure of the valve by means of x-ray examination; and a description of the principles of operation, the tensile strength of the components and the behavior before MRI. ISO 7197 (International..., 2006) also presents the hydrodynamic tests that are essential for evaluation of the shunt and its respective components. ISO 7197 (International..., 1997) discusses in great detail the requirements for the construction of the testing rigs that are employed in the performance of the tests, which are given below:

1) Opening and closing pressure of the system: this determines the necessary pressure for starting and stopping the flow, considering the resistance of the catheter and the resistive elements present inside the neurological valves to control the flow of the liquid. ISO 7197 (International..., 1997) indicates the use of testing rigs, illustrated in Figure 1, composed of the following: an infusion pump (1) with the ability to maintain the flow of distilled water (fluid) at a rate of $20 \mathrm{ml} / \mathrm{h}$; a reservoir with distilled water at $37 \pm 2{ }^{\circ} \mathrm{C}$, maintained at that level with a tolerance of $\pm 2 \mathrm{~mm}$; a neurological valve (3); a manometer graduated in $\mathrm{mm}$ (2), with an accuracy of $0.5 \mathrm{~mm}$ and an inner diameter of $2.5 \mathrm{~mm}$. The pressures shown on the pressure gauge where the flow enters through the neurological valve must be registered as well as the cut-off pressure after switching off the pump and waiting for the flow to stabilize.

2) Characteristics of pressure-flow: these enable the relationship between the pressure gradient and the shunt flow to be obtained. The use of the test apparatus shown in Figure 1 permits evaluation of the performance of the shunt, through the infusion of distilled water, at flow rates of 50, 40, $30,20,10$ and $5 \mathrm{ml} / \mathrm{h}$ and collects the respective pressures indicated on the manometer.

3) Shunt system performance regarding the reflow: this indicates that the neurological valve must prevent backflow exceeding $0.2 \mathrm{ml}$ for 5 minutes with opposing pressures of 100 and $500 \mathrm{~mm}$ $\mathrm{H}_{2} \mathrm{O}$ in a column against the fluid flow sensed in the valve.

The requirements of these standards are fundamental to the study and development of neurological valves and shunts for the treatment of hydrocephalus; however, this work emphasizes the hydrodynamic tests presented in items 1,2 , and 3 based on the importance they have for the performance evaluation of shunts, which makes it possible to develop or improve products that offer better control in the drainage of cerebrospinal fluid.
ISO 7197 (International..., 1997) mentions that different resources can be employed for the implementation of flow and pressure collection systems, provided that they meet the requirements of the standard and provide better technical conditions in carrying out the activities. Several researchers utilize hydrodynamic testing rigs to evaluate the performance of the neurological valves and components present in shunts.

Drake and Sainte-Rose (1994) present examples of testing rigs that measure the pressure-flow interface and the opening and closing pressures similarly to that presented in Figure 1, but without the manometer and with the addition of pressure transducers, and with different principal elements, using a water reservoir at a constant level to replace the infusion pump and an electronic balance to quantify the flow; in addition, they present another testing rig with a pressure transducer and a flow meter to avoid the use of the balance. Camilo Pinto (2005) comments on testing rigs used for hydrodynamic tests in shunts and presents various forms of graphical presentations of the obtained results.

To perform pressure-flow tests and evaluate the valves' opening and closing, Allin et al. (2008) and Czosnyka et al. (1997; 2002), employed a testing rig containing pressure transducers, a water reservoir at a constant level, an infusion pump, a pulse generator and an electronic balance, with an additional apparatus that allowed the testing of shunts in a pressurized environment.

Suriano et al. (2012), determined the relative pressure-flow rate of a shunt on a testing rig to show that changes to the original diameter and length of the peritoneal catheter, during installation of the implant or surgical revision, change the hydrodynamic behavior of the drainage system and can induce errors in the

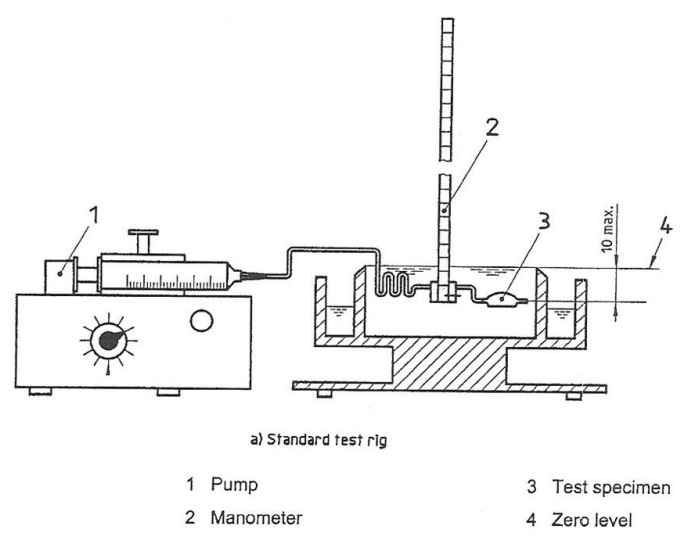

Figure 1. Testing rig for the determination of opening and closing pressures of the neurological valve according to ISO 7197 (International..., 1997). 
clinical evaluation of the patient by the neurosurgeon. Changes in the shunt components can modify the neurological valve pressure class and interfere in the treatment of hydrocephalus.

Allin et al. (2008), Czosnyka et al. (1997, 2002) and Suriano et al. (2012) presented their results with statistical analyses. However, there was no evidence of evaluation of the testing rigs used in the tests or the analysis of experimental uncertainties.

Evaluation of the apparatus used in hydrodynamic testing is fundamental so that the experimental uncertainties will not lead to misinterpretation of the shunt system's performance.

This study presents the assessment of a hydrodynamic testing rig, built according to ISO 7197 (International..., 1997, 2006), related to uncertainties in the precision of the flow and opening and closing pressure measurements, the pressure-flow relationship and the resistance to reflux of the neurological valves.

\section{Methods}

Among the hydrodynamic tests, determination of the relationship between pressure and flow in shunts is usually the most frequently reported item in scientific publications, and it indicates the behavior of the flow of cerebrospinal fluid facing the pressure gradient in the VPS.

The CSF flow (Q) through the internal drainage system is governed by the pressure gradient between the ventricle and the peritoneal area $(\Delta \mathrm{P})$ and the resistance $(\mathrm{R})$ imposed by the neurological valve and the cylindrical ducts through which the fluid passes (Czosnyka et al., 1997; Drake and Sainte-Rose, 1994; Gusmão et al., 2000; Maset et al., 2009; Pudenz and Foltz, 1991), as shown in Equation 1.

$Q=\frac{\Delta P}{R}$

The gradient of the system pressure is under the influence of various pressures forms: intraventricular pressure (IVP) at the entrance of the ventricular catheter; intra-abdominal pressure (IAP) in the output of the peritoneal catheter; hydraulic pressure due to the difference in height between the ends of the catheters $(\mathrm{H})$ caused by the fluid column; and the closing pressure of the valve $(\mathrm{CP})$, which represents the pressure responsible for interrupting the flow.

Intraventricular pressure and hydrostatic height facilitate the fluid run-off, while the intra-abdominal pressure offers resistance to the flow; therefore, one can define the pressure gradient as shown in Equation 2 (Czosnyka et al., 1997; Gusmão et al., 2000; Maset et al., 2009; Pudenz and Foltz, 1991).
$Q=\frac{I V P+H-I A P-C P}{R}$

The system resistance (R), according to Maset et al. (2009), is a sum of the resistances of the ventricular catheter, the neurological valve and the peritoneal catheter.

According to ISO 7197 (International..., 1997, 2006), applying different flows in the shunts and observing the respective pressures makes it possible to determine the relationship between the pressure gradient and flow (Equation 1). The same testing rig can be used to determine the opening and closing pressures of the neurological valves apart from their resistance to CSF reflux.

The testing rig developed to perform hydrodynamic tests for the opening and closing pressure of neurological valves, the pressure-flow characteristics and the behavior of the shunt in the presence of reflux, as illustrated in Figure 1, was developed in accordance with ISO 7197 (International..., 1997), with the addition of a pressure sensor with an amplifier circuit and a signal acquisition board connected to the computer to record the gauge pressure.

Figure 2 presents the test apparatus, which consists of an infusion pump (1) SyringePump, NE1000 model, manufactured by New Era Pump Systems Inc., Farmingdale, NY, EUA, programmed by the computer, that infuses distilled water through a tube connected to a graduated and calibrated manometer with an inner diameter of $2.5 \mathrm{~mm} \mathrm{(2),} \mathrm{and} \mathrm{a} \mathrm{neurological}$ valve (3) and peritoneal catheter (4). A glass reservoir (5), containing distilled water (6) maintained at a temperature of $37 \pm 2{ }^{\circ} \mathrm{C}$ by a temperature controller (7) and verified by a calibrated thermometer (8). The collection of values is performed by a pressure sensor (9) produced by GE NovaSensor, model NPC-100 $(5 \mu \mathrm{V}$ sensitivity and linearity of measurement between

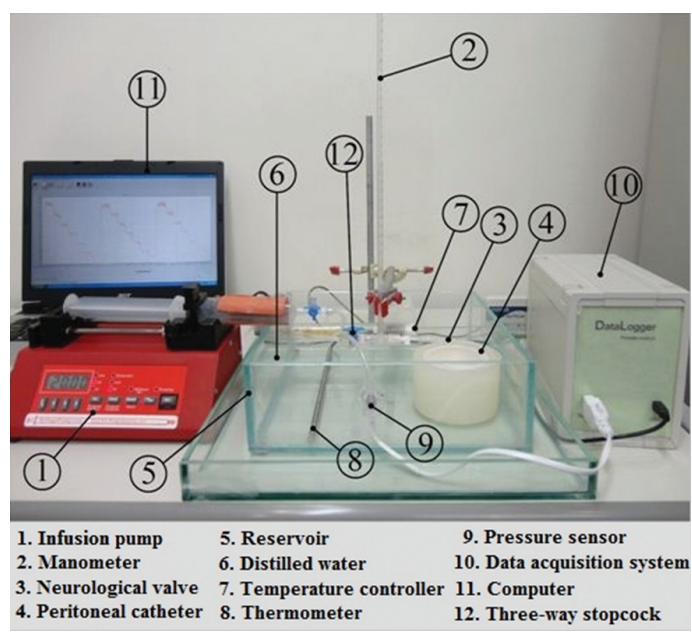

Figure 2. Testing rig for neurological valves. 
-400 and $1300 \mathrm{~mm} \mathrm{H}_{2} \mathrm{O}$ ), a data acquisition system (10) and a computer (11). A three-way stopcock (12) drives the fluid to the sensor, which measures the pressure in the system. The calibrated pressure gauge (2) allows the pressure to be read for monitoring the automated collection system.

The assessment of the accuracy of flow rate and pressure measurement by the testing rig is based on evaluation of the experimental uncertainties of the variables involved in the hydrodynamic tests, determination of the opening and closing pressures, the pressure-flow relationship and the reflux resistance of two commercial products. The assessment is also based on evaluation of the functioning of the testing rig, within the context of its application, by comparing the experimental results with information submitted by the manufacturers because companies use their own testing rigs as the standard and test their products for registration at ANVISA to guarantee the quality of the VPS.

The main variables involved in hydrodynamic tests are flow and pressure; thus, the reliability of the equipment used, such as the infusion pump, the pressure sensor connected to the automated system of data collection and the heater of the distilled water in the reservoir, are essential to ensure accurate results.

Infusion pump calibration was performed using a Marte electronic balance, model AS2000C, with a resolution of $0.01 \mathrm{~g}$. Flows of 5, 10, 20, 30, 40 and $50 \mathrm{ml} / \mathrm{h}$ were infused for a period of 5 minutes, and the variation of water mass over time was compared. The test was repeated 5 times for each flow. The stability of the infusion pump throughout the process was evaluated by the variation of infusion at $20 \mathrm{ml} / \mathrm{h}$ for 1 hour.

The pressure measuring system, consisting of a pressure transducer (GE Sensor, model NTC-100), an amplifier and a signal acquisition board (myPCLab, version 1.22, manufactured by NOVUS), was calibrated using a manometer. The pressures between 20 and $500 \mathrm{~mm} \mathrm{H}_{2} \mathrm{O}$ and the registry of values of each $10 \mathrm{~mm} \mathrm{H}_{2} \mathrm{O}$ between the 20 and $200 \mathrm{~mm} \mathrm{H}_{2} \mathrm{O}$ marks were compared, as were every $50 \mathrm{~mm} \mathrm{H}_{2} \mathrm{O}$ between 200 and $500 \mathrm{~mm} \mathrm{H}_{2} \mathrm{O}$. The procedure was performed 5 times. The characterization of the sensor behavior considered the possibility of hysteresis with the loading and unloading of the pressure in the pressure range between 0 and $500 \mathrm{~mm} \mathrm{H}_{2} \mathrm{O}$ and register values of every $50 \mathrm{~mm} \mathrm{H}_{2} \mathrm{O}$. The procedure was performed 3 times.

The water heating system was assessed with a temperature acquisition system (myPCLab, version 1.22 , manufactured by NOVUS) placed in the fluid in the reservoir, into which the VPS system is inserted.

The uncertainties involved in the execution of the experiments and the confidence interval of the results determine the system's reliability. Thus, the uncertainties are determined for the equipment that provides the values for the key variables involved: flow and pressure.

The testing rig, illustrated in Figure 2, was used to test a neurological valve manufactured by Integra, the Contour-Flex Valve System Small model, and a valve manufactured by Ventura Biomedica, the Synchrony model, Cello series; both are classified as medium pressure with their respective peritoneal catheters. The purpose of this study was to carry out tests for the opening and closing pressures, the pressure-flow relationship and reflux-resistance and to compare the results with the information submitted by the manufacturers.

The opening and closing pressures were registered after filling the tubing, catheter and valve with distilled water and after verifying the absence of bubbles in the system, by infusing the fluid to a flow rate of 20 $\mathrm{ml} / \mathrm{h}$ and simultaneously collecting the pressure data for 5 minutes, after interrupting the infusion for 5 additional minutes. The procedure was performed 5 times for each shunt.

The pressure-flow relationship of the tested products was evaluated using the same test apparatus; however, distilled water was infused at flow rates of $50,40,30,20,10$ and $5 \mathrm{ml} / \mathrm{h}$ for five minutes; the pressures were collected between each flow. The test was carried out 5 times for each shunt.

The reflux flow was raised by reversing the position of the neurological valve to the preceding position on the three-way stopcock and the manometric tube. The infusion pump was programmed to infuse the distilled water until the tube indicated a pressure of $100 \mathrm{~mm} \mathrm{H}_{2} \mathrm{O}$, after which the tube connected to the valve was disconnected for volumetric quantification related to the fall of the pressure gauge, which occurs when there is return of the liquid through the valve in the anterograde direction to flow. The procedure was also performed at a pressure of $500 \mathrm{~mm} \mathrm{H}_{2} \mathrm{O}$ as indicated on the manometer tube, and both tests were carried out for 5 minutes and repeated 5 times.

The results obtained from the tests were compared with the values reported by the manufacturers in the manuals for the tested products.

\section{Results}

The testing rig for the hydrodynamic tests of the neurological valves was built entirely according to ISO 7197 (International..., 1997).

The calibration of the infusion pump for flows of $5,10,20,30,40,50$ and $60 \mathrm{ml} / \mathrm{h}$ was accomplished with the aid of a precision electronic balance and an automated data collection system. The obtained flow rates are presented in Table 1.

The stability of the infusion pump can be defined as the ability to maintain constant flow for long periods of time. The stability test was conducted for 
the reference flow of $20 \mathrm{ml} / \mathrm{h}$, with a duration of 1 hour, significantly longer than the 5-minute duration of the valve pressure test acquisition. The average flow rate observed in the stability test of the infusion pump was $19.92 \mathrm{ml} / \mathrm{h}$, with a maximum observed variation of only $1 \%$, showing that the equipment presents good flow stability.

The pressure measurement system consists of a pressure transducer-type strain gage with an electronic circuit and an amplifier with a board (A/D) for signal acquisition. The calibration of the electronic pressure measuring system was accomplished with the aid of a manometer and a graduated liquid column. The system was calibrated with a resolution of $0.5 \mathrm{~mm}$ in the range between 20 and $500 \mathrm{~mm} \mathrm{H}_{2} \mathrm{O}$, and the behavior is shown in Figure 3. Tests were also carried out to verify if hysteresis occurs in the electronic pressure measuring system by applying increasing pressures between 0 and $500 \mathrm{~mm} \mathrm{H}_{2} \mathrm{O}$ and observing the decrease to $0 \mathrm{~mm} \mathrm{H}_{2} \mathrm{O}$. The tests did not indicate the occurrence of appreciable hysteresis in the measurement range.

The system used to heat the distilled water in the reservoir where the neurological valve is submerged was evaluated for a 2-hour period; the temperature was maintained at $37.1 \pm 0.4^{\circ} \mathrm{C}$. The bath temperature remained within the values established by ISO 7197 (International..., 2006) $\left(37 \pm 2^{\circ} \mathrm{C}\right)$.

According to Abernethy et al. (1985) and Moffat (1988), uncertainties related to the equipment used on the testing rig and used for measurement should be considered. A random uncertainty of 2 times the standard deviation was adopted to obtain a probability of $95 \%$ and an uncertainty of the balance of $0.2 \mathrm{~g}$ or $0.2 \mathrm{ml}$ equivalent; the infusion pump uncertainties were calculated for the flows of 10 and $50 \mathrm{ml} / \mathrm{h}$ and were close to the extreme values of the flow used to calibrate the infusion pump, resulting in an uncertainty of less than $1 \%$. The uncertainties of the pressure sensor, considering an uncertainty of the pressure gauge equal to $0.5 \mathrm{~mm} \mathrm{H}_{2} \mathrm{O}$, were lower than $6 \%$ for $20 \mathrm{~mm} \mathrm{H}_{2} \mathrm{O}$ and below $4 \%$ for all other pressures.

The use of the testing rig for the hydrodynamic test of a Contour-Flex valve from Integra and a Synchrony valve from the Cello series by Ventura Biomedica, both classified as medium-pressure valves, enabled the determination of the opening and closing pressures (Table 2), the pressure-flow characteristics (Table 3, Figures 4 and 5) and the resistance to reflux for comparison with the values provided by the manufacturers in the manuals for their respective products.

During the test for the determination of the opening and closing pressures, in the first few seconds, the valves resisted the flow, and the pressure at the manometer tube increased until the moment at which the beginning of the flow caused changes in the pressure behavior, indicating the opening pressure of the valve. After 5 minutes of interruption of the infusion, the closing pressure, responsible for the interruption of the flow, was registered. The obtained values are shown in Table 2.

The results of the tests of the pressure-flow relationship of the valves indicate the performance of these devices and are presented in Table 3.

Figures 4 and 5 present the upper control limit (UCL) and the lower control limit (LCL), according to the information in the manuals of the tested products (Integra, 2013; Ventura, 2013). The test results show that the valves used are within the limits recommended by the manufacturers.

The Contour-Flex neurological valve presented an average reflux of 0.004 and $0.005 \mathrm{ml}$ during the 5 minutes of the test at active pressures of 100 and $500 \mathrm{~mm} \mathrm{H}_{2} \mathrm{O}$ column in the output of the valve; the

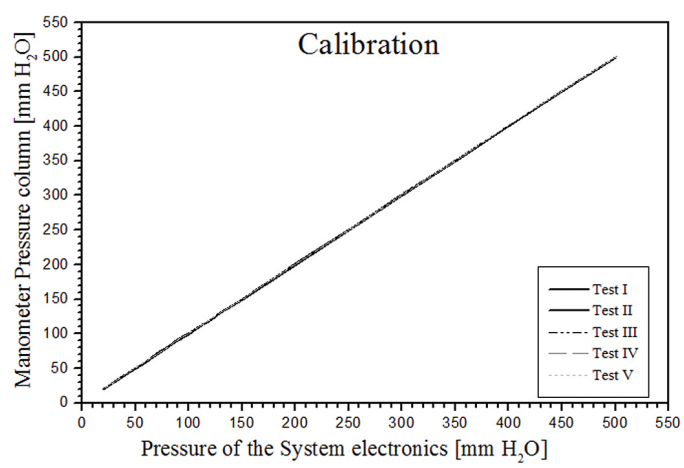

Figure 3. Values from calibration of the pressure sensor.

Table 1. Values of the programmed flow rates in the infusion pump compared to the values obtained with the aid of electronic balance.

\begin{tabular}{|c|c|c|c|c|c|c|c|c|}
\hline \multirow{2}{*}{$\begin{array}{l}\text { Flow } \\
(\mathrm{ml} / \mathrm{h})\end{array}$} & \multicolumn{5}{|c|}{ Test } & \multirow{2}{*}{ Average } & \multirow{2}{*}{$\begin{array}{l}\text { Standard } \\
\text { deviation }\end{array}$} & \multirow{2}{*}{$\begin{array}{l}\text { Systematic } \\
\text { uncertainty }\end{array}$} \\
\hline & $\mathbf{A}$ & B & $\mathrm{C}$ & D & $\mathbf{E}$ & & & \\
\hline 60 & 59.94 & 60.10 & 59.76 & 59.04 & 59.34 & 59.64 & 0.44 & 0.36 \\
\hline 50 & 49.50 & 49.62 & 50.49 & 49.98 & 49.56 & 49.83 & 0.41 & 0.17 \\
\hline 40 & 39.54 & 39.84 & 39.94 & 39.66 & 39.66 & 39.73 & 0.16 & 0.27 \\
\hline 30 & 29.40 & 29.94 & 29.70 & 29.70 & 29.88 & 29.72 & 0.21 & 0.28 \\
\hline 20 & 20.10 & 19.98 & 19.92 & 19.74 & 19.80 & 19.91 & 0.14 & 0.09 \\
\hline 10 & 9.78 & 9.78 & 9.72 & 9.78 & 9.90 & 9.79 & 0.07 & 0.21 \\
\hline 5 & 5.34 & 4.86 & 4.88 & 4.95 & 5.23 & 5.05 & 0.22 & 0.05 \\
\hline
\end{tabular}


Table 2. Opening and closing values of the neurological valves.

\begin{tabular}{|c|c|c|c|c|}
\hline \multirow{3}{*}{ Test } & \multicolumn{2}{|c|}{ Contour-Flex } & \multicolumn{2}{|c|}{ Cello } \\
\hline & Opening & Closing & Opening & Closing \\
\hline & \multicolumn{2}{|c|}{$\left(\mathbf{m m ~ H _ { 2 }} \mathrm{O}\right)$} & \multicolumn{2}{|c|}{$\left(\mathbf{m m ~ H _ { 2 }} \mathrm{O}\right)$} \\
\hline 1 & 71 & 51 & 27 & 24 \\
\hline 2 & 51 & 56 & 26 & 27 \\
\hline 3 & 50 & 50 & 31 & 24 \\
\hline 4 & 59 & 52 & 24 & 25 \\
\hline 5 & 53 & 52 & 36 & 24 \\
\hline Average & 56.8 & 52.2 & 28.8 & 24.8 \\
\hline Standard deviation & 8.7 & 2.3 & 4.8 & 1.3 \\
\hline
\end{tabular}

Table 3. Results of Flow x Pressure.

\begin{tabular}{ccccc}
\hline \multirow{2}{*}{ Flow $(\mathbf{m l} / \mathbf{h})$} & \multicolumn{2}{c}{ Contour-Flex } & \multicolumn{2}{c}{ Cello } \\
\cline { 2 - 5 } & Average & Standard deviation & Average & Standard deviation \\
\hline 60 & 129.12 & 2.56 & 136.06 & 4.89 \\
50 & 120.16 & 2.09 & 121.74 & 4.18 \\
40 & 110.98 & 2.06 & 108.12 & 3.41 \\
30 & 101.52 & 1.82 & 93.70 & 2.81 \\
20 & 91.18 & 1.59 & 77.68 & 3.25 \\
10 & 78.90 & 1.12 & 58.62 & 3.11 \\
5 & 70.34 & 1.25 & 42.72 & 6.88 \\
\hline
\end{tabular}

Cello valve presented 0.002 and $0.007 \mathrm{ml}$ reflux at the same pressures.

\section{Discussion}

ISO 7197 (International..., 2006) corresponds to the current regulations for neurological implants for treatment of hydrocephalus; however, ISO 7197 (International..., 1997) discusses in great detail the rigs for hydrodynamic testing, and the information remains valid after reviewing this standard.

For the specific case of the infusion pump, systematic uncertainties (or accuracy) are obtained from the difference between the values programmed into the infusion pump and the average value of the mass variation measured by the electronic balance for a predetermined time interval of data acquisition. The time interval for acquiring the data for the mass variation of the balance was 0.237 seconds, which was sufficient to obtain a statistically significant average value once the transmission rate of the balance reached 253 values per minute. The work of Camilo Pinto (2005) shows, in detail, how determining the time span depends on the transmission rate of the balance data. In turn, random uncertainties (or precision) are represented by the standard deviation of the readings. The smallest value of systematic uncertainty $(0.05 \mathrm{ml} / \mathrm{h})$ was observed for the lowest flow rate, and the largest value $(0.36 \mathrm{ml} / \mathrm{h})$ for the

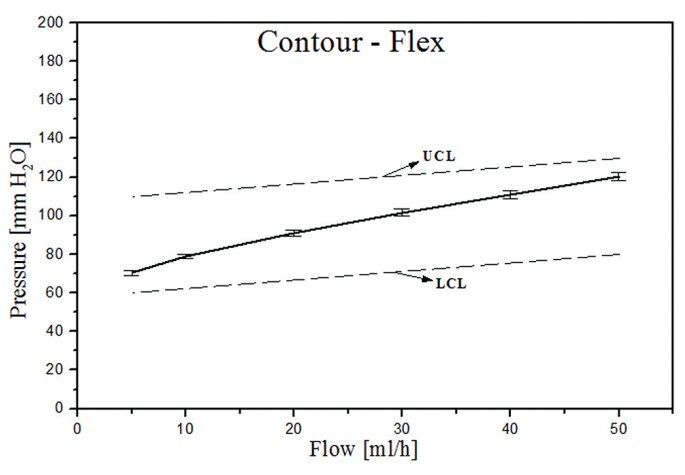

Figure 4. Behavior of pressure $\mathrm{x}$ flow for the Contour Flex valve.

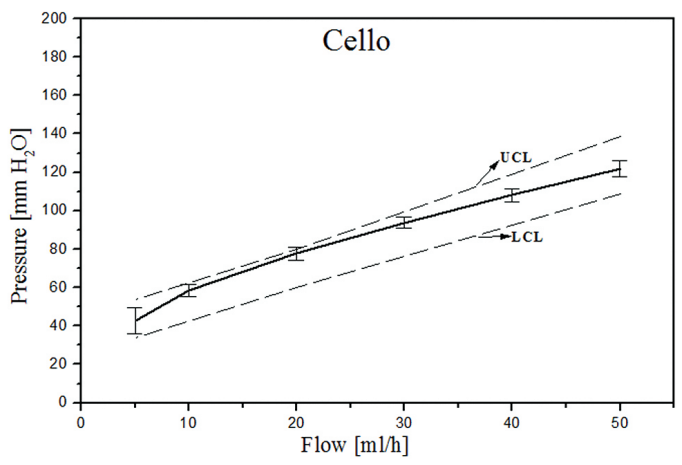

Figure 5. Behavior of pressure $\mathrm{x}$ flow for the Cello valve. 
highest flow rate. The average value of the systematic error percentage is less than $1 \%$.

The flow rate of $20 \mathrm{ml} / \mathrm{h}$ may be considered as a reference due to the proximity of this value to the flow rate usually observed in healthy adults. A systematic uncertainty of just $0.09 \mathrm{ml} / \mathrm{h}$ or $0.45 \%$ was observed for the flow rate of $20 \mathrm{ml} / \mathrm{h}$, i.e., the infusion pump showed an uncertainty between the programmed value and the obtained value equivalent to $0.09 \mathrm{ml} / \mathrm{h}$, demonstrating good accuracy in performing its task of carrying out the tests, mainly at the flow rate of $20 \mathrm{ml} / \mathrm{h}$, which determines the operating pressure of the shunt.

The pressure measurement system presented absolute systematic uncertainties relating to $0.20 \mathrm{~mm}$ $\mathrm{H}_{2} \mathrm{O}$ for most of the reference pressures; at some pressures, no systematic uncertainties were present, and the largest systematic uncertainty was $1 \mathrm{~mm} \mathrm{H}_{2} \mathrm{O}$, at a pressure of $300 \mathrm{~mm} \mathrm{H}_{2} \mathrm{O}$, which also presented the largest random uncertainty of $1.41 \mathrm{~mm} \mathrm{H}_{2} \mathrm{O}$ (standard deviation). Most random uncertainties were approximately $0.50 \mathrm{~mm} \mathrm{H}_{2} \mathrm{O}$. The pressure measurement behavior did not display sharp hysteresis through the sensor, when loading and unloading pressures in the range between 0 and $500 \mathrm{~mm} \mathrm{H}_{2} \mathrm{O}$ column were considered. The occurrence of significant hysteresis in the sensor leads to the registration of different pressure values in the shunt, which affects the results obtained in the hydrodynamic tests and the performance evaluation of valve systems.

Knowing the opening and closing pressures of the neurological valves in the shunts is of great importance because they represent the resistance to the beginning and interruption of the flow, respectively. Neurological valves with opening pressure values higher than the value of their operating pressure (pressure related to the flow of $20 \mathrm{ml} / \mathrm{h}$ ) cause excessive resistance at the beginning of the flow and an increase in ventricular volume; and closing pressures close to $0 \mathrm{~mm} \mathrm{H}_{2} \mathrm{O}$ provide low resistance to the blockage of drainage and a decrease in ventricular volume. The values obtained for the opening and closing pressures in the hydrodynamic testing are within the acceptable range to avoid disturbances of the ventricular volume. Because the manufacturers' manuals did not include the values for the opening and closing pressures, it was not possible to compare those values with the results obtained using the testing rig.

The neurological valves tested for their resistance to reflux presented results within the limit of $0.04 \mathrm{ml} /$ min control that has been established by ISO 7197 (International..., 2006), so that no injury can be caused to the patient regarding the return of fluid to the ventricle due to bodily positions that might contribute to the occurrence of reflux; for example, when the patient remains with the head positioned below the peritoneal area.
ISO 7197 (International..., 2006) indicates that the manufacturer should specify the classes of operation or functioning of neurological valves: for example, extra low, low, medium or high pressure, and their respective ranges of pressure gradients should relate to the $20 \mathrm{ml} / \mathrm{h}$ flow that corresponds, approximately, to the CSF flow in the human body.

The Contour-Flex valve is available in three functioning classes: namely, low, medium and high pressure, with variations between 20 and 60, 70 and 120 , and 130 and $180 \mathrm{~mm} \mathrm{H}_{2} \mathrm{O}$, respectively. The Cello valve offers five classes of operation: extra low, low, medium, normal, or high pressure, with variations between 10 and 30,35 and 55, 60 and 80, 90 and 110 and 125 and $145 \mathrm{~mm} \mathrm{H}_{2} \mathrm{O}$. Hydrodynamic testing of a Contour-Flex valve in a testing rig that logs the pressure as $70 \mathrm{~mm} \mathrm{H}_{2} \mathrm{O}$ and has an experimental uncertainty exceeding $14.3 \%$ compromises the reliability of the results; for example, if the real hydrodynamic behavior of the valve is low pressure, and if the prescribed pressure is $120 \mathrm{~mm}$ $\mathrm{H}_{2} \mathrm{O}$, an uncertainty in reading the pressure of $8.5 \%$ can hide the behavior of a high pressure valve. In a similar way, for the limits of pressure variation of a medium pressure Cello valve, uncertainties greater than $8.5 \%$ and $12.5 \%$ in the pressure recording can in fact indicate hydrodynamic behavior of low and normal pressure valves, respectively.

The testing rig for hydrodynamic tests was suitable for performing the tests for opening and closing, resistance to reflux and pressure-flow characteristics of neurological valves.

The testing rig used in the study showed total uncertainties of less than $1 \%$ for fluid infusion, $2.1 \%$ for pressures between 70 and $90 \mathrm{~mm} \mathrm{H}_{2} \mathrm{O}$, and $4 \%$ for most pressures obtained, and do not compromise the results and evaluation of in vitro performance of the valve and neurological components present in a ventricle-peritoneal shunt.

The testing rig for the hydrodynamic tests in neurological valves was built in accordance with ISO 7197 (International..., 1997) and uses a pressure measurement system similar to those described by Allin et al. (2008), Czosnyka et al. (1997, 2002) and Drake and Sainte-Rose (1994). This work presents an assessment of the rigs used in the tests and an analysis of experimental uncertainties, which are not often presented in scientific publications that describe apparatus for testing neurological valves. Ignorance of the experimental uncertainties of testing rigs can lead to inaccurate results and erroneous assessments of the items tested, such as incorrect determination of the operating pressure, the pressure-flow relationship and the opening and closing pressures of the shunt, as well as the behavior of internal elements of the neurological valve that prevent the reflux of CSF. 
The accuracy of the flow enforcement and pressure measurement in neurological valve testing rigs is fundamental to provide reliable results for the analysis of shunt performance and to aid in improving neurological valve components; however, information about the uncertainties of the experimental apparatus used is not usually provided in publications.

\section{References}

Abernethy RB, Benedict RP, Dowdell RB. ASME mensurement uncertainty. Journal of Fluids Engineering. 1985; 107:161-4. http://dx.doi.org/10.1115/1.3242450

Allin DM, Czosnyka M, Richards HK, Pickard JD, Czosnyka $\mathrm{ZH}$. Investigation of the hydrodynamic properties of a new MRI-resistant programmable hydrocephalus shunt. Cerebrospinal Fluid Research. 2008; 5(8):1-11.

Aschoff A, Kremer P, Hashemi B, Kunze S. The scientific history of hydrocephalus and its treatment. Neurosurgical Review. 1999; 22:67-93. http://dx.doi.org/10.1007/ s101430050035

Camilo Pinto JR. Simulação hidrodinâmica e caracterização experimental de mecanismo anti-sifão em sistemas de drenagem externa de líquido cefalorraquidiano [dissertação]. Ilha Solteira: Universidade Estadual Paulista; 2005. 96 p.

Czosnyka M, Czosnyka Z, Whitehouse H, Pickard JD. Hydrodinamic properties of hydrocephalus shunts: United Kingdom shunt evaluation laboratory. Journal of Neurology, Neurosurgery and Psychiatry. 1997; 62:43-50. http://dx.doi. org/10.1136/jnnp.62.1.43

Czosnyka Z, Czosnyka M, Richards HK. Pickard, JD. Laboratory testing of hydrocephalus shunts: conclusion of the U.K. shunt evaluation programe. Acta Neurochirurgica. 2002; 114:525-38. http://dx.doi.org/10.1007/ s00701-002-0922-9

Drake JM, Sainte-Rose C. The shunt book. Paris: Blackwell Science; 1994.

Gusmão S, Silveira RL, Cabral Filho G, Arantes A. Aplicações clínicas da hidrodinâmica na derivação ventrículo-peritoneal. Arquivos Brasileiros Neurocirurgia. 2000; 19(4):179-83.

Integra. Contour-flex valve: instruction for use - contourflex valve and shunt systems. Plainsboro: Integra NeuroSciences; 2013.

International Organization for Standardization. ISO 7197: Neurological implants: sterile, single-use hydrocephalus shunts and components. Switzerland; 1997.
International Organization for Standardization. ISO 7197: Neurological implants: sterile, single-use hydrocephalus shunts and components. Switzerland; 2006.

Lavinio A, Harding S, Boogaard FVD, Czosnyka M, Smielewski P, Richards HK, Pickard JD, Czosnyka ZH. Magnetic field interactions in adjustable hydrocephalus shunts. Journal Neurosurgery Pediatrics. 2008; 2:222-8. http://dx.doi.org/10.3171/PED/2008/2/9/222

Maset AL, Camilo Pinto JR, Andrade JR, Xavier VEF. Considerações hidrodinâmicas sobre derivação liquórica: tecnologia de válvulas - primeira geração. Arquivos Brasileiros de Neurocirurgia. 2009; 28(3):87-96.

Moffat RJ. Describing the uncertainties in experimental results. Experimental Thermal and Fluids Science. 1988; 1:317. http://dx.doi.org/10.1016/0894-1777(88)90043-X

Penn RD, Basati S, Sweetman B, Guo X, Linninger A. Ventricle wall movements and cerebrospinal fluid flow in hydrocephalus. Journal Neurosurgery. 2011; 15:159-64. http://dx.doi.org/10.3171/2010.12.JNS10926

Pople IK. Hydrocephalus and shunts: what the neurologist should know. Journal of Neurological Neurosurgery Psychiatry. 2002; 73:117-22.

Pudenz RH, Foltz EL. Hydrocephalus: overdrainage by ventricular shunts. Surgical Neurology. 1991; 35:200-12. http://dx.doi.org/10.1016/0090-3019(91)90072-H

Schimer M. Neurocirurgia. São Paulo: Santos Editora; 1995.

Sotelo J, Izurieta M, Arriada N. Treatment of hydrocephalus in adults by placement of an open ventricular shunt. Journal Neurosurgery. 2001; 94:873-9. http://dx.doi.org/10.3171/ jns.2001.94.6.0873

Suriano IC, Maset AL, Fontolan TA, Monteiro R, Camilo JR, Andrade JR, Freitas DG, Rodrigues TP, Paz DA, Cavalheiro S. Considerações hidrodinâmicas sobre a derivação liquórica $\mathrm{V}$ : alterações nos dimensionamentos originais do sistema valvular no momento do implante alteram suas características hidrodinâmicas e induzem a erros de avaliação clínica pelo neurocirurgião. Arquivos Brasileiros de Neurocirurgia. 2012; 31(4):207-18.

Ventura. Synchrony hydrocephalus shunt: manual de instruções - valve for hydrocephalus synchrony série cello. São José do Rio Preto: Ventura Biomédica; 2013.

Wood JH. Neurobiology of cerebrospinal fluid. v. 2. New York: Plenum Press; 1980. http://dx.doi.org/10.1007/9781-4684-1039-6

\title{
Authors \\ José Ricardo Camilo Pinto*, João Manuel Domingos de Almeida Rollo \\ Programa de Pós-graduação Interunidades em Bioengenharia, Universidade de São Paulo - USP, \\ Av. Trabalhador São-carlense, 400, CEP 13566-590, São Carlos, SP, Brasil.
}

\author{
Angelo Luiz Maset, José Ricardo Andrade, Bruna Monieli Mancini, Geovânia Marquini Laurentino Pereira, \\ Ricardo César Barbosa \\ Departamento de Hidrodinâmica, Ventura Biomédica, São José do Rio Preto, SP, Brasil.
}

